\title{
Philosophiques
}

\section{L’ironie auctoriale : une approche gricéenne est-elle possible ?}

\section{Anne Reboul}

Volume 35, numéro 1, printemps 2008

Les valeurs de l'ironie (1) et le scepticisme à l'âge classique (2)

URI : https://id.erudit.org/iderudit/018234ar

DOI : https://doi.org/10.7202/018234ar

Aller au sommaire du numéro

\section{Éditeur(s)}

Société de philosophie du Québec

ISSN

0316-2923 (imprimé)

1492-1391 (numérique)

Découvrir la revue

\section{Citer cet article}

Reboul, A. (2008). L'ironie auctoriale : une approche gricéenne est-elle possible ? Philosophiques, 35(1), 25-55. https://doi.org/10.7202/018234ar

\section{Résumé de l'article}

Grice a proposé une analyse de l'ironie fondée sur les implicatures, selon laquelle les énoncés ironiques produisent une implicature par antiphrase. Cette thèse, qui suit l'analyse rhétorique classique, la transpose simplement du registre sémantique au pragmatique, ce qui ne suffit pas à répondre à la question de savoir comment l'auditeur saisit l'interprétation par antiphrase, ou pourquoi le locuteur dit une chose quand il signifie l'inverse. L'analyse antiphrastique ne dit pas non plus comment on doit rendre compte des énoncés ironiques qui ne sont pas des assertions. Les analyses contemporaines de l'ironie, comme celles de Sperber et Wilson en termes d'écho, et de Currie en termes de feintise -, ne rencontrent pas les mêmes difficultés. On les présente en général comme capables de rendre compte des cas " centraux " d'ironie et comme incompatibles entre elles.

Dans le présent article, je montre que les deux analyses s'appliquent au même ensemble d'exemples et qu'en fait certaines critiques de Currie contre l'analyse échoique ne sont pas valides. De plus il y a un ensemble d'exemples d'énoncés ironiqques que l'on ne peut pas analyser en termes de feintise. Donc aucune des deux analyses n'est assez générale. Pour finir, je propose une analyse selon laquelle les énoncés ironiques montrent (plutôt qu'ils ne disent) un comportement, une croyance ou un raisonnement déraisonnable, et je plaide pour une analyse gricienne, basée non pas sur l'implicature par antiphrase, mais sur la signification non naturelle et la reconnaissance de la double intention du locuteur. Cette analyse est compatible avec l'analyse échoïque et avec celle en termes de feintise, tout en étant plus générale.
Ce document est protégé par la loi sur le droit d'auteur. L’utilisation des services d'Érudit (y compris la reproduction) est assujettie à sa politique d'utilisation que vous pouvez consulter en ligne.

https://apropos.erudit.org/fr/usagers/politique-dutilisation/ 


\title{
L'ironie auctoriale: une approche gricéenne est-elle possible?
}

\author{
ANNE REBOUL \\ Institut des sciences cognitives \\ Lyon, France
}

À ce propos, je m'étonne dirai-je de l'ingratitude ou de la paresse des mortels, qui tous me rendent un culte assidu, jouissent volontiers de mes bienfaits, et dont pas un seul, depuis tant de siècles, ne s'est montré pour célébrer avec gratitude les louanges de Folie, alors qu'on a vu des gens perdre leur huile et leur sommeil pour vanter dans des discours soigneusement travaillés, les Busiris, les Phalaris, les fièvre quartes, les mouches, les calvities et autres fléaux de ce genre.

ERASME, Éloge de la folie, III.

\begin{abstract}
RÉSUMÉ. - Grice a proposé une analyse de l'ironie fondée sur les implicatures, selon laquelle les énoncés ironiques produisent une implicature par antiphrase. Cette thèse, qui suit l'analyse rhétorique classique, la transpose simplement du registre sémantique au pragmatique, ce qui ne suffit pas à répondre à la question de savoir comment l'auditeur saisit l'interprétation par antiphrase, ou pourquoi le locuteur dit une chose quand il signifie l'inverse. L'analyse antiphrastique ne dit pas non plus comment on doit rendre compte des énoncés ironiques qui ne sont pas des assertions. Les analyses contemporaines de l'ironie, comme celles de Sperber et Wilson en termes d'écho, et de Currie - en termes de feintise -, ne rencontrent pas les mêmes difficultés. On les présente en général comme capables de rendre compte des cas «centraux» d'ironie et comme incompatibles entre elles.
\end{abstract}

Dans le présent article, je montre que les deux analyses s'appliquent au même ensemble d'exemples et qu'en fait certaines critiques de Currie contre l'analyse échoique ne sont pas valides. De plus il y a un ensemble d'exemples d'énoncés ironïques que l'on ne peut pas analyser en termes de feintise. Donc aucune des deux analyses n'est assez générale. Pour finir, je propose une analyse selon laquelle les énoncés ironiques montrent (plutôt qu'ils ne disent) un comportement, une croyance ou un raisonnement déraisonnable, et je plaide pour une analyse gricienne, basée non pas sur l'implicature par antiphrase, mais sur la signification non naturelle et la reconnaissance de la double intention du locuteur. Cette analyse est compatible avec l'analyse échoïque et avec celle en termes de feintise, tout en étant plus générale.

ABSTRACT. - Grice proposed an implicature-based account of irony, according to which ironical utterances give rise to an antiphrasis implicature. This view, which followed the classical rhetorical account of irony, merely transported it from the semantic to the pragmatic domain, which is clearly not enough to answer the questions which the antiphrasis account triggers, i.e., the explanation of how the hearer 
recovers the antiphrasis interpretation, or of why the speaker should say something when she means exactly the reverse. A final, and devastating, criticism is, quite simply, that not all ironical utterances are assertions and, hence, that the antiphrasis account does not easily apply to them. What is more, some ironical utterances, perhaps most of them, do not at all trigger an antiphrasis. Contemporary accounts of irony, such as those proposed by Sperber and Wilson — the echoic account - or by Currie - the pretence account —, do not meet with the same difficulties. They are generally presented as being able to account for "central" examples of irony and as incompatible..

In the present paper, I will show that the echoic and the pretence accounts, far from being incompatible, seem to be applicable to exactly the same set of examples, and that, in fact, some of the strictures levelled by Currie against the echoic account are not in fact valid criticism. Additionally, there are quite a lot of examples of ironical utterances which are not susceptible of an account in terms of echo or pretence. Thus, it seems that neither account can serve as a general account of irony. I finally propose an account in terms of ironical utterances showing (rather than saying) an unreasonable behaviour, belief or reasoning on the part of the target of the irony and plead for a Gricean account, based not on an antiphrasis implicature, but on meaningNN and the recognition of the double-barrelled intention of the speaker. This, clearly, is compatible with the echoic or pretence accounts, though more general than either.

\section{Introduction}

Les approches courantes de l'ironie, qu'elles soient philosophiques - comme celle de Currie (2006) — ou « linguistiques » — comme celle de Sperber et Wilson (1981, 1995) —, s'appuient, comme le font les analyses philosophiques ou linguistiques de la métaphore, sur des exemples «standard ". Dans le cas de la métaphore, cela conduit à se concentrer sur des exemples comme 1 à 3 :

1. Caroline est une princesse.

2. Bill est un bulldozer ${ }^{1}$.

3. Juliette est le soleil².

Mis à part le fait que cette liste semble restreinte si l'on considère que la métaphore est une figure de rhétorique dont on a pu dire qu'elle était produite en plus grande quantité par les poissonnières parisiennes du XVII ${ }^{\mathrm{e}}$ siècle qu'à l'Académie française de la même époque (cf. Dumarsais, 1988), il devrait être évident au premier coup d'œil que la composition syntaxique uniforme des exemples ci-dessus n'augure rien de bon pour la généralité ou le caractère robuste de l'analyse proposée, quelle qu'elle soit. Que fait-on, par exemple, de cas comme :

1. (1) et (2) sont empruntées à Wilson et Carston (2006).

2. Cet exemple, tiré de Roméo et Juliette, est habituellement censé tenir le rôle d'une métaphore créative par contraste avec (1) et (2), qui sont plus ou moins des métaphores passées dans l'usage courant (des «métaphores mortes» ou inertes). 


\section{KING CLAUDIUS}

[...] There is something in his soul

O'er which his melancholy sits on brood,

And I doubt the hatch and the disclose

Will have some danger; [...].

(W. Shakespeare, Hamlet, III, 1).

[Il y a quelque chose dans son âme

que couve sa mélancolie

et je crains que son éclosion et son apparition

ne soit dangereuse.]

5. Le sommeil de la raison engendre des monstres.

(F. Goya, 1797, Titre du frontispice des Caprices).

\section{THE LOVE SONG OF J. ALFRED PRUFROCK}

The yellow fog that rubs its back upon the window-panes,

The yellow smoke that rubs its muzzle on the window-panes

Licked its tongue into the corners of the evening,

Lingered upon the pools that stand in drains,

Let fall upon its back the soot that falls from chimneys,

Slipped by the terrace, made a sudden leap,

And seeing that it was a soft October night,

Curled once about the house, and fell asleep.

(T.S. Eliot)

[Le brouillard jaune qui se frotte le dos contre les carreaux des fenêtres, la fumée jaune qui frotte son museau aux carreaux de la fenêtre

a léché avec sa langue les coins de la soirée,

s'est penchée sur les flaques qui reposent sous les gouttières,

a laissé tomber sur son dos la suie qui tombe des cheminées,

a glissé sur la terrasse, a fait un bond soudain,

et voyant que c'est une douce nuit d'octobre,

s'est couchée en rond sur la maison et s'est endormie.]

De fait, les analyses courantes n'ont pas grand chose à en dire. La diversité même des énoncés ironiques, qui présentent une grande variété de formes syntaxiques, et correspondent à divers actes de langage (assertion, question, ordre, exclamation, etc.), devrait décourager toute tentative de restreindre, de façon similaire, l'ironie à un petit nombre d'exemples assertifs. C'est néanmoins exactement ce qui se produit et l'exemple central habituel dans les articles sur l'ironie est:

7. Pierre: Quel beau jour pour un pique-nique!

[Ils partent en pique-nique et il pleut à torrents.]

Marie: C'est vraiment un beau jour pour un pique-nique ${ }^{3}$ !

3. Sperber et Wilson (1995) et Currie (2006) utilisent cet exemple. 
Cette utilisation d'exemples soi-disant standard pour des figures de rhétorique implique que l'on obtient une analyse qui peut être parfaitement adaptée à de tels exemples, mais qui échoue de façon évidente pour des exemples moins "standard ». Cela explique, par exemple, pourquoi l'analyse de la métaphore en termes de concepts ad hoc (cf. Wilson et Carston, 2006), qui peut fonctionner de façon satisfaisante pour (1) et (2), et peut-être même pour (3), ne semble pas s'appliquer à (4), (5) ou (6). Qu'en est-il de l'ironie ?

De fait, il semble clair que les mêmes remarques s'appliquent à l'identique à l'ironie et cela suggère que l'ironie (de même, probablement, que d'autres figures de rhétorique) n'est pas une catégorie bien définie. En effet, de la même façon que l'on peut aligner des exemples qui paraissent limites pour la métaphore ${ }^{4}$, on peut sans difficulté trouver des exemples qui sont limites pour l'ironie :

8. Un religieux de l'ordre de Saint Benoît, Ernold le Pingouin, effaça à lui seul quatre mille manuscrits grecs et latins, pour copier quatre mille fois l'évangile de Saint Jean. Ainsi furent détruits en grand nombre les chefs-d'œuvre de la poésie et de l'éloquence artistique. Les historiens sont unanimes à reconnaître que les couvents pingouins furent le refuge des lettres au moyen âge (A. France, L'Île des pingouins, 107-108).

9. La belle princesse recommanda le secret à ses dames sur ce qu'elles avaient vu. Elles le promirent toutes, et en effet le gardèrent un jour entier (Voltaire, Le Taureau blanc, 242).

10. Le plaisant de l'éducation actuelle, c'est qu'on n'apprend rien aux jeunes filles qu'elles ne doivent oublier bien vite dès qu'elles seront mariées (Stendhal, De l'amour, 244).

11. Il est du véritable amour comme de l'apparition des esprits: tout le monde en parle, mais peu de gens en ont vu (La Rochefoucauld, Maximes, 76).

Bien que (8) soit sans doute un exemple «standard" d'ironie - quoi que cela veuille dire - , on voit (9) et (10) comme plutôt moins "standard ", alors que (11) serait probablement un cas limite, c.-à-d. que certains diraient, alors que d'autres nieraient, que c'est de l'ironie. En d'autres termes, il est douteux que les figures de rhétorique en général, et la métaphore et l'ironie en

4. Par exemple, est-ce que (a) est une métaphore? :

(a) Love Poems

'Remember' is a lost cry on a wind:

A bollow nothing-heard,

Most memorable, in a deaf night

That does not heed.

(Lawrence Durrell)

[ «Souviens-toi» est un cri perdu dans le vent

un vide inaudible,

plus mémorable, dans une nuit sourde

Qui ne l'entend pas.] 
particulier, soient des catégories bien délimitées. Cela, à la vérité, a été noté par Sperber et Wilson $(1981,298)^{5}$ : «L'existence d'une catégorie unifiée de l'ironie ne devrait pas être considérée comme évidente. » Ils insistent plus fortement sur le même point en 1995 (240) : «Nous doutons fortement qu'il y ait soit un sous-ensemble bien défini d'attitudes ironiques soit un sous-ensemble bien défini d'énoncés ironiques qui les expriment. [...] L'ironie n'est pas une espèce naturelle. »

Si c'est bien le cas, cela soulève un doute supplémentaire : s'il n'y a pas de catégorie bien délimitée de l'ironie — en abandonnant la métaphore à son sort - alors on peut supposer que les cas «standard "sont identifiés sur la base d'une notion intuitive que la plupart des gens reconnaîtraient comme de l'ironie, alors que, pour d'autres exemples, moins « standard ", la proportion des gens disposés à y voir de l'ironie diminuerait progressivement au fur et à mesure de leur distance avec les cas « standard ${ }^{6} »$. Cette remarque apparemment innocente a une conséquence importante : il est douteux qu'il y ait une caractéristique unique, à la fois commune à et centrale pour tous les énoncés ironiques, ce qui, incidemment, suffit à jeter le doute sur toutes les analyses existantes de l'ironie, des analyses classiques en termes de contre-vérité et d'antiphrase, au plus récentes en termes d'énoncés échoïques (Sperber et Wilson 1995) ou de feintise (pretence: Currie 2006). De fait, cela suggère que l'ironie est une question de ressemblance de famille, plutôt que (d'un ensemble) de condition(s) nécessaire(s) et suffisante(s).

Si c'est bien le cas, on devrait se rappeler qu'un des enseignements de la notion wittgensteinienne de ressemblance de famille est que ce qui unit les membres d'une famille donnée n'est pas tant une (unique) caractéristique qu'ils partagent que le fait que chacun d'entre eux partage avec un autre une caractéristique qui peut n'être partagée par aucune autre paire de membres de la famille, y compris des paires dont l'un ou l'autre d'entre eux est un membre. En d'autres termes, bien qu'il puisse y avoir une caractéristique partagée par tous les membres de la famille, qui, de ce fait, serait une caractéristique diagnostique, il pourrait tout aussi bien ne pas y en avoir une. Ainsi, quand des instances d'un concept sont unies par une ressemblance de famille, l'existence d'une caractéristique unique est contingente, pour ne pas dire assez peu plausible. De ce fait, le poids de la preuve est, effectivement, lourd pour quelque tentative qui essaie de proposer une analyse «unifiée » de l'ironie ${ }^{8}$.

5. Toutes les citations tirées d'ouvrages en anglais (y compris les exemples littéraires) sont traduits par mes soins. Les caractères italiques ou majuscules sont dans le texte d'origine, sauf s'il est dit explicitement qu'ils sont de mon cru.

6. Cela semble, de fait, suggéré par Currie $(2006,131)$ : «Ce qu'une théorie de l'ironie doit faire est de montrer qu'elle a les ressources nécessaires pour caractériser, de façon intuitivement acceptable, l'endroit où est situé le centre de gravité de l'ironie, et de mesurer, de nouveau de façon intuitivement acceptable, les distances depuis cet endroit à d'autres endroits sur la carte. »

7. Pretence n'a pas d'équivalent en français. Kevin Mulligan suggère feintise, utilisé par Bergson (1959).

8. De façon évidente, la même chose serait vraie — de fait est vraie — pour la métaphore... 
L'analyse gricéenne (Grice, 1989) tombe-t-elle sous les mêmes critiques ? Elle approche le problème en termes d'implicature conversationnelle (prima facie compatible avec les remarques ci-dessus), s'appuyant sur l'idée que la fausseté évidente des énoncés ironiques déclenche une implicature conversationnelle avec un contenu antiphrastique, une conclusion tout à fait consistante avec l'analyse classique de l'ironie (cf. la discussion ci-dessous, $\mathbb{S} 2$ ). Comme Sperber et Wilson $(1981,296)$ le notent : «La proposition de Grice libérerait la théorie sémantique du problème de définir la signification figurative et de dériver cette signification dérivative de l'énoncé. Cependant, ces problèmes ne sont pas résolus par le simple fait de les transférer du domaine sémantique au domaine pragmatique. » Cela paraît effectivement une critique parfaitement valide. Dès lors, quelle analyse gricéenne pourrait être possible pour l'ironie, si, comme c'est clairement le cas, une analyse en termes d'implicature ne remplit pas cette tâche?

De fait, je suis totalement en accord avec le rejet d'une analyse gricéenne en termes d'implicature'. Cependant, je pense qu'une analyse gricéenne alternative, s'appuyant non sur la notion d'implicature et sur le principe de coopération, mais sur la notion de signification non naturelle (signification $\mathrm{NN}$ ), n'est pas seulement possible, mais nécessaire. De fait, il se pourrait, étant donné ce qui a été dit plus haut, que ce soit la seule façon de faire sens de l'ironie comme phénomène interprétatif, plutôt que comme une catégorie linguistique bien définie.

\section{L'analyse anti-phrastique de l'ironie}

L'analyse classique de l'ironie en fait une anti-phrase: en d'autres termes, un énoncé ironique comme (7) - reproduit ci-dessous en (12) - bien qu'il dise C'est vraiment un beau jour pour un pique-nique! veut en fait dire Ce n'est vraiment pas un beau jour pour un pique-nique ! ${ }^{10}$ :

12. Pierre: Quel beau jour pour un pique-nique!

[Ils partent en pique-nique et il pleut à torrents]

Marie: C'est vraiment un beau jour pour un pique-nique!

Cette analyse est cependant très peu satisfaisante, comme le remarquent Sperber et Wilson $(1981,295)$ :

Un énoncé ironique est traditionnellement analysé comme disant littéralement une chose et signifiant de façon figurative l'inverse. Ainsi la remarque ironique

9. Et, comme nous allons bientôt le voir, cette analyse n'est pas seulement inacceptable parce qu'elle balaie le problème — supposément — sémantique sous le tapis pragmatique, mais parce qu'il n'est pas clair que l'ironie déclenche de façon habituelle une interprétation anti-phrastique. (cf. $\left.\int 2\right)$.

10. (7) pourrait, de façon peut-être plus plausible, être interprété comme «C’est un jour abominable pour un pique-nique. » Cela ne constitue cependant pas une objection réelle à l'analyse antiphrastique, dans la mesure où «C'est un jour abominable pour un pique-nique » peut difficilement être interprété autrement que comme contredisant «C'est un beau jour pour un pique-nique.» 
Quel temps magnifique! aurait la signification figurative "Quel temps épouvantable! », et ainsi de suite. Une théorie sémantique explicite conçue pour incorporer une telle analyse devrait fournir, d'abord, une définition de la signification figurative; ensuite, un mécanisme pour dériver la signification figurative d'une phrase; et enfin, une base quelconque qui permette d'expliquer pourquoi les énoncés figuratifs existent: pourquoi un locuteur devrait préférer la remarque ironique Quel temps magnifique! à sa contrepartie littérale Quel temps épouvantable! qui, selon cette analyse, signifie exactement la même chose. C'est parce qu'elles ne fournissent aucune réponse à de telles questions que les analyses sémantiques traditionnelles de l'ironie échouent au bout du compte.

Cela est complètement approprié comme critique d'une partie de la théorie selon laquelle l'ironie est une antiphrase, c.-à-d. que cela indique de façon correcte qu'une telle analyse n'explique rien en elle-même. En d'autres termes, c'est une critique bien fondée basée sur le fait que l'analyse antiphrastique ne remplit aucune condition d'adéquation explicative. Elle ne dit rien cependant de son adéquation descriptive ${ }^{11}$ : en d'autres termes, est-il vrai que les énoncés ironiques déclenchent habituellement des interprétations antiphrastiques ? Et si ce n'est pas exact, qu'est-ce qui explique, non seulement l'existence de cette analyse, mais sa longévité ${ }^{12}$ ?

Une des raisons pour lesquelles l'analyse antiphrastique est, ou semble être, si robuste pourrait être liée à une caractéristique "standard» fréquemment attribuée (mais de façon moins proéminente) à l'ironie, c.-à-d., la contre-vérité. La contre-vérité n'est pas une simple fausseté, mais plutôt un type particulier de fausseté : une fausseté évidente, parce que la situation même de la communication falsifie clairement la proposition exprimée (comme en [12] ci-dessus), ou parce que la proposition est contradictoire, soit de facon directe (comme en [8]), soit de façon indirecte, à travers ses implications les plus accessibles (comme en [13]) :

13. On a remarqué qu'à Constance il y avait eu sept cent dix-huit filles pour le service du concile qui fit brûler si dévotement Jean Hus et Jérôme de Prague (Voltaire, L'Homme aux 40 écus, 164).

Cette analyse semble justifiée, au moins en apparence, en ce que la même phrase exactement, C'est vraiment un beau jour pour un pique-nique! peut être produite soit ironiquement, comme en (12), soit sérieusement, comme en (14):

14. Pierre: Quel beau jour pour un pique-nique!

[Ils partent en pique-nique et le soleil brille.]

Marie: C'est vraiment un beau jour pour un pique-nique!

11. Sur la distinction entre adéquation explicative et adéquation descriptive, cf. Chomsky (1957).

12. L'analyse antiphrastique remonte à la Grèce classique et a survécu jusqu'à maintenant (cf. Perrin, 1996). 
Comme la seule différence entre (12) et (14) semble être que le premier — ironique - est une contre-vérité, alors que le second — sérieux — n'en est pas une, il semble naturel de supposer qu'il y a un lien direct entre interpréter un énoncé comme ironique et l'identifier comme une contre-vérité. De fait, Grice a franchi ce pas dans son analyse originelle lorsqu'il a suggéré que c'est le fait même que les énoncés ironiques sont des contre-vérités qui déclenche l'implicature antiphrastique.

Cependant, Sperber et Wilson $(1981,1995)$ ont fait la critique additionnelle que les énoncés ironiques ne sont pas toujours des assertions : ils correspondent à différents types d'actes de langage auxquels il n'est pas sûr que l'analyse en termes de contre-vérité puisse s'appliquer de façon correcte. Pour l'instant, j'aimerais évaluer si l'antiphrase caractérise réellement l'interprétation ironique et, si ce n'est pas le cas, si une des raisons de l'attrait de cette analyse (pour les énoncés ironiques assertifs) ne tient pas à l'occurrence fréquente de la contre-vérité dans les énoncés ironiques (assertifs).

Comme Sperber et Wilson (1981) le disent justement dans leur critique de l'analyse antiphrastique citée ci-dessus, les raisons pour lesquelles un locuteur qui veut communiquer la proposition $\neg p$ devrait utiliser dans ce but un énoncé qui exprime la proposition $p$ (ou vice versa) ne sont pas claires. Mais, de façon plus pertinente, la raison pour laquelle, dans des cas de contre-vérités comme (12) où la proposition exprimée est évidemment et immédiatement falsifiée, le locuteur voudrait informer son interlocuteur de ce qui devrait déjà lui être évident n'est pas plus claire. Incidemment, le locuteur, par l'énonciation de (12), violerait la maxime de sincérité, mais si l'implicature de (12) était effectivement Ce n'est pas un beau jour pour un pique-nique, il aurait aussi violé la maxime de pertinence. En d'autres termes, une analyse de l'ironie en termes d'implicature ne semble pas prometteuse étant donné qu'elle ne restaure pas le principe de coopération, mais en aggrave la violation. Qui plus est, pourquoi le locuteur voudrait-il communiquer une vérité évidente?

Supposons, pour permettre la discussion, que tous les énoncés ironiques assertifs soient des contre-vérités ${ }^{13}$. Pouvons-nous expliquer pourquoi l'analyse antiphrastique, qui paraît à première vue si peu satisfaisante, a été aussi populaire? Je pense qu'on le peut, si l'on suppose que les interlocuteurs appliquent habituellement le principe de charité à l'interprétation des énoncés ; en d'autres termes, les interlocuteurs essaient d'interpréter ce que disent les locuteurs de façon à éviter de leur attribuer une irrationalité massive. Si les énoncés ironiques sont généralement des contre-vérités (comprises comme des faussetés évidentes), il serait plus charitable de supposer que les locuteurs:

13. Cela, comme nous allons bientôt le voir, n'est pas le cas. Mais il faut reconnaître que les énoncés ironiques assertifs sont très souvent des contre-vérités, ce qui suffit à expliquer la popularité de l'analyse antiphrastique. 
- ne croient pas que les contre-vérités sont vraies;

- ne croient pas que leurs interlocuteurs croiraient de telles contrevérités (c.-à-d. que les locuteurs ne mentent pas).

Autrement dit, étant donné que les locuteurs ne croient pas la proposition exprimée vraie et qu'ils ne mentent pas, la solution la plus simple peut sembler être l'analyse antiphrastique. Cela, bien évidemment, ne signifie pas que l'analyse antiphrastique soit adéquate descriptivement ou explicativement ${ }^{14}$, mais seulement que, malgré son inadéquation évidente, sa longévité est compréhensible.

Revenons-en à la contre-vérité : est-ce vraiment une caractéristique de tous les énoncés ironiques? Bien que ce soit une caractéristique fréquente, il faut bien reconnaître qu'elle n'est pas universelle, comme le montre l'exemple suivant ${ }^{15}$ :

15. Rien n'était si beau, si leste, si brillant, si bien ordonné que les deux armées. [...] Les canons renversèrent d'abord à peu près six mille hommes de chaque côté; ensuite la mouqueterie ôta du meilleur des mondes environ neuf à dix mille coquins qui en infectaient la surface. La baïonnette fut aussi la raison suffisante de la mort de quelques milliers d'hommes. Le tout pouvait bien se monter à une trentaine de mille âmes. Candide, qui tremblait comme un philosophe, se cacha du mieux qu'il put pendant cette boucherie héroïque.

Enfin, tandis que les deux rois faisaient chanter des Te Deum chacun dans son camp, il prit le parti d'aller raisonner ailleurs des effets et des causes (Voltaire, Candide, 14).

Dans ce cas particulier, on ne voit pas comment défendre de façon raisonnable une analyse en termes de contre-vérité : la proposition tandis que les deux rois faisaient chanter des Te Deum chacun dans son camp décrit deux événements, les services religieux par lesquels chaque roi célèbre sa victoire dans son propre camp. Bien évidemment, ils ne peuvent pas tous deux avoir gagné la bataille. Cependant, ce n'est pas ce que la proposition dit : ce qu'elle dit est, indiscutablement, vrai ${ }^{16}$. De fait, on peut dire que, si ce ne l'était pas, l'énoncé ne serait pas ironique. Ainsi, (15) contredit la thèse (forte) selon laquelle tous les énoncés ironiques sont des contre-vérités.

Cependant, cet exemple est parfaitement compatible avec une hypothèse moins exigeante proposée par Currie $(2006,119)$ : «Le point important est que l'ironie représente - et donc peut représenter de façon erronée — sa cible comme déraisonnable d'une façon ou d'une autre, ou au moins comme échouant à un critère saillant de raisonnabilité quelconque. » J'y reviendrai.

14. Comme on l'a sans doute compris, je pense qu'elle n'est ni l'un ni l'autre.

15. C'est un exemple très fréquemment utilisé. Il devrait avoir été utilisé pour montrer l'inadéquation de la plupart des analyses. Les raisons pour lesquelles on a pu l'utiliser pour les justifier me sont incompréhensibles.

16. Dans ce cas spécifique, bien évidemment, vrai dans la fiction. Ce qui n'est pas vrai dans la fiction est que chacun des rois a gagné. Mais c'est un autre problème. 
Enfin, le fait que certains énoncés ironiques ne soient pas des contrevérités, étant donné la restriction habituelle à des énoncés ironiques «standard ", ne contredit pas l'explication donnée ci-dessus de la popularité de l'analyse antiphrastique. Venons-en à deux analyses plus contemporaines, celles de Sperber et Wilson $(1981,1995)$ et de Currie (2006).

\section{Préliminaire: deux stratégies pour introduire du vague dans les théories de l'ironie}

Les difficultés qu'implique la recherche d'une analyse "unitaire » de l'ironie n'ont pas échappé aux théories contemporaines. Ainsi, la plupart, si ce n'est la totalité, des analyses de l'ironie commencent fortes et finissent faibles, en suivant deux stratégies mutuellement compatibles:

1. La première consiste à affaiblir la caractéristique même qui est supposée être centrale dans l'ironie, en la faisant passer d'une caractéristique assez fortement contrainte ${ }^{17}$ à une caractéristique moins fortement contrainte ${ }^{18}$; c'est, par exemple, la stratégie suivie par Sperber et Wilson, qui passent d'une définition absolue de la mention — d'abord formulée en termes philosophiques - à une définition relative, plus adaptée à des phénomènes du langage naturel, comme l'ironie;

2. La seconde consiste à appliquer l'analyse, non pas à la proposition effectivement exprimée dans l'énoncé, mais à une quelconque implicitation ultérieure de cette proposition. C'est clairement le cas de l'approche de Perrin (1996), qui essaie de maintenir toutes les analyses en les appliquant d'abord à la proposition exprimée dans l'énoncé, et ensuite en les appliquant à des implicitations de plus en plus lointaines de l'énoncé. Par exemple, l'analyse antiphrastique est préservée comme s'appliquant, non pas à la proposition exprimée, mais à une implicitation ultérieure : en (15), l'antiphrase s'appliquerait non pas à la proposition exprimée (les deux rois ont fait chanter des Te Deum dans leur camp respectif), ni à une implicitation immédiatement accessible (chacun des deux camps célébrait sa victoire), ni à une implicitation ultérieure (chacun des deux rois pensait qu'il avait gagné), mais à quelque chose qui est le contenu de la pensée rapportée dans l'implicitation précédente (J'ai gagné, pensé par chaque roi) ${ }^{19}$ [U21].

Le résultat, dans les deux cas, est le même : l'analyse devient suffisamment vague pour être maintenue malgré les contre-exemples (supposés apparents). Cela, il faut le noter, n'est pas nécessairement une critique destructive : comme je l'ai indiqué ci-dessus (cf. $\mathbb{S} 1$ ), l'ironie est une catégorie interprétative, ce qui implique qu'elle n'est pas bien délimitée et qui, si on suppose

17. Dans le sens où elle peut être précisément définie et est une caractéristique absolue.

18. Dans le sens où elle ne peut pas être précisément définie et où c'est une caractéristique relative plutôt qu'absolue.

19. Cette analyse particulière n'a pas été proposée par Perrin (1996), mais, d'après d'autres analyses qu'il propose, il ne semble pas faire de doute que c'est la stratégie qu'il suit. 
qu'elle a une caractéristique universelle, nécessite probablement un peu de souplesse interprétative en ce qui concerne la possession par un énoncé spécifique quelconque de la caractéristique en question. Les deux stratégies esquissées ci-dessus ne sont rien d'autre que deux façons de répondre à cette nécessité. Je voudrais maintenant décrire les deux théories, la façon dont elles affaiblissent explicitement (Sperber et Wilson) ou implicitement (Currie) leurs analyses originelles pour tenir compte du caractère intrinsèquement vague de la catégorie des énoncés ironiques, et, finalement, pour montrer que, au bout du compte, leurs stratégies échouent.

\section{Sperber et Wilson : les énoncés ironiques comme des énoncés échoïques}

J'ai déjà fait allusion plusieurs fois ci-dessus à l'approche de l'ironie proposée par Sperber et Wilson, notamment en rappelant leur critique de la théorie antiphrastique de l'ironie. Leur approche a d'abord été proposée dans un article en $1981^{20}$, où l'analyse était d'abord formulée en termes de mention. La distinction (philosophique) entre usage et mention est basée sur des exemples comme les suivants :

16. Mon chat a trois pattes.

17. «Chat» est un mot de quatre lettres.

Clairement, en (16), le mot chat a sa pleine fonction référentielle normale. En (17), il ne l'a pas: il n'y a pas de chat auquel il est fait référence. Dans le premier cas, le mot chat est utilisé, alors que dans le second, il est mentionné. Bien que les exemples classiques de la distinction usage/mention concernent le plus souvent des mots, la distinction peut aussi être appliquée à des phrases complètes ou à des propositions (au sens grammatical). Suivant cette piste, Sperber et Wilson $(1981,303)$ disent :

L'USAGE d'une expression implique la référence à ce à quoi l'expression réfère ; la MENTION d'une expression implique la référence à l'expression elle-même. [...] Quand l'expression mentionnée est une phrase complète, elle n'a pas la force illocutionnaire qu'elle aurait de façon standard dans un contexte où elle serait utilisée $e^{21}$.

Cette dernière remarque met en lumière deux points importants :

- Les énoncés ironiques sont très souvent interprétés comme ironiques relativement à un contexte (par exemple, il serait difficile de voir la dernière phrase de (8) comme ironique si elle n'apparaissait pas à la suite des phrases précédentes qui construisent le contexte approprié);

20. J'utiliserai à la fois l'article de 1981 et la version ultérieure de l'analyse échoïque donnée dans leur ouvrage (Sperber et Wilson 1995, chapitre 4, $\mathbb{\$} 9$ ). Les différences entre les deux versions sont relativement limitées et ne sont pas pertinentes pour la discussion présente.

21. Majuscules des auteurs. 
- Les énoncés ironiques sont très souvent interprétés comme n'ayant pas la force illocutionnaire que leur forme linguistique devrait entraîner, et cet échec, selon la théorie classique des actes de langage (cf. Searle, 1969), vient du fait qu'il est souvent évident que la condition de sincérité n'est pas satisfaite par le locuteur $^{22}$ dans un énoncé ironique.

Bien que la distinction usage/mention ait été considérée pendant longtemps comme absolue, Sperber et Wilson $(1981,303)$ ont défendu une distinction plus faible et relative: "Dans les langues naturelles, les mentions prennent une diversité de formes, dont certaines pourraient être des cas intermédiaires, tombant quelque part entre l'usage et la mention. » Autrement dit, bien que la distinction usage/mention puisse être absolue selon la vision épurée de la philosophie, son application au langage naturel la rend relative plutôt qu'absolue, alignant potentiellement les cas limite de mention et les cas limite d'ironie. L'ironie est encore réduite à une sous-catégorie de la mention échoïque, c.-à-d. à la mention d'un énoncé précédent quelconque.

L'affaiblissement de la définition de la mention, la rendant relative plutôt qu'absolue, est un exemple de la première stratégie décrite ci-dessus. Cependant, Sperber et Wilson utilisent aussi la seconde stratégie, comme le montre la citation suivante: "Il y a aussi des cas où ce à quoi on fait écho n’est pas une proposition exprimée par un énoncé, mais une pensée attribuée par le locuteur à son interlocuteur» (Sperber et Wilson 1981, 308). Qui plus est, "un énoncé échoïque n’a pas à interpréter une pensée précisément attribuable : il peut faire écho à la pensée d'une certaine sorte de personne, ou des gens en général » (Sperber et Wilson, 1995, 238). En d'autres termes, l'écho peut être implicite, mais il peut additionnellement être l'écho d'une pensée (dont on suppose qu'elle est privée) et ne pas être attribué à une personne, à un groupe spécifique. Cela semble une caractérisation très faible de l'ironie, et il est difficile de voir exactement comment elle pourrait satisfaire une contrainte fixée par les auteurs eux-mêmes :

Pour l'interlocuteur, comprendre un tel énoncé implique tout à la fois de réaliser que c'est un cas de mention plutôt que d'usage, mais aussi de reconnaître l'attitude du locuteur envers la proposition mentionnée. Cette interprétation dans sa totalité dépend de cette double reconnaissance (Sperber et Wilson 1981, 308).

Il y a ici une difficulté supplémentaire qui tient au fait que, contrairement à l'analyse de Sperber et Wilson, certains exemples supposés «standard » d'ironie, comme (15), reproduit ci-dessous en (18) ne semblent pas du tout être des échos :

18. Rien n'était si beau, si leste, si brillant, si bien ordonné que les deux armées. [...] Les canons renversèrent d'abord à peu près six mille hommes de

22. Cela lie de façon lâche l'analyse de la mention tout à la fois à l'analyse en termes de contre-vérité et à l'analyse en termes de feintise : les deux points sont communs ou au moins compatibles avec ces analyses alternatives. 
chaque côté ; ensuite la mouqueterie ôta du meilleur des mondes environ neuf à dix mille coquins qui en infectaient la surface. La baïonnette fut aussi la raison suffisante de la mort de quelques milliers d'hommes. Le tout pouvait bien se monter à une trentaine de mille âmes. Candide, qui tremblait comme un philosophe, se cacha du mieux qu'il put pendant cette boucherie héroïque.

Enfin, tandis que les deux rois faisaient chanter des Te Deum chacun dans son camp, il prit le parti d'aller raisonner ailleurs des effets et des causes (Voltaire, Candide, 14).

Voici l'analyse de Sperber et Wilson ${ }^{23}$ : «Voltaire fait écho à des affirmations faites par les rois ennemis. Dans la mesure où ces affirmations se contredisent l'une l'autre, il est clair que s'il est minimalement alerte mentalement, il ne peut pas les avaliser toutes les deux» (Sperber et Wilson 1995, 241). Cela est cependant très dérivatif : la proposition ironique décrit deux événements, c.-à-d. les Te Deum chantés dans les deux camps sur les ordres des rois respectifs, et elle ne fait écho aux affirmations de l'un ou l'autre roi que sous l'hypothèse qu'un énoncé décrivant une action intentionnelle fait de ce fait même écho à l'état mental de l'agent. Cela semble aller bien loin, et faire de chaque description d'un événement intentionnel un écho potentiel de l'intention correspondante.

Au cas où cela semblerait une critique trop sévère de l'analyse proposée par Sperber et Wilson, je voudrais juste faire remarquer que celle-ci ne fait pas plus mal que l'analyse antiphrastique classique ou, comme nous allons bientôt le voir, que l'autre analyse contemporaine dominante, c.-à-d. l'analyse en termes de feintise. Qui plus est, malgré tous les problèmes que rencontre l'analyse échoïque, elle met en lumière deux facteurs cruciaux de l'ironie :

- Les énoncés ironiques ont une cible;

- Ils communiquent une attitude — le plus souvent négative - envers la cible ou envers les processus mentaux de la cible tels qu'ils sont illustrés par l'énoncé ironique.

\section{Currie: l'ironie comme feintise}

La théorie de l'ironie proposée par Currie est bien résumée par l'affirmation suivante :

L'ironie prétend asserter ou questionner ou justifier et ce faisant exprime une attitude envers ceux qui feraient, diraient ou justifieraient de cette façon, ou envers

23. En toute justice, leur analyse est basée sur une traduction erronée de l'exemple de Voltaire, empruntée directement d'un ouvrage (en anglais) sur l'ironie: "When all was over and the rival kings were celebrating their victory with Te Deum in their respective camps... " plutôt que sur la version plus fidèle suivante: "At last, while the two kings had Te Deum sung in their respective camps..." 
des gens, des actions et des attitudes que la feintise amène par ailleurs à l'esprit. Cette expression n'est pas nécessairement un cas de dire. De fait, l'ironie peut se passer du langage (Currie 2006, 113).

La question centrale pour une telle approche est la définition exacte de ce qu'est la feintise. Bien que Currie ne donne pas de définition explicite, il donne quelques indications. Selon lui :

la feintise qui est fondamentale dans l'ironie n'est pas une feintise d'action; c'est une feintise d'être. En prétendant asserter ou quoi que ce soit d'autre, on prétend être une certaine sorte de personne - une personne avec une vision restreinte ou défective d'une façon ou d'une autre du monde ou d'une partie du monde (Currie 2006, 116).

Et il ajoute: «Mais l'ironie est [...] limitée par la contrainte qu'elle cible seulement des perspectives d'une certaine sorte: celles auxquelles nous pouvons appliquer un standard de raisonnabilité » (Currie 2006, 118).

Comme le note Currie, cette analyse peut être vue - on l'a dit comme très semblable, si ce n'est entièrement réductible à l'analyse échoïque de Sperber et Wilson. Il rejette fortement cette vision des choses, affirmant que « ces approches ${ }^{24}$ sont différentes [...] et la théorie échoïque échoue à rendre compte de quelque chose qui distingue l'ironie d'autres formes d'énoncés soidisant échoïques » (Currie 2006, 112), à savoir la feintise.

Si l'on suppose pour l'instant que Currie a raison et que la théorie de la feintise n'est pas réductible à la théorie échoïque, où, exactement, réside la différence? Une suggestion naturelle serait que la notion même d'écho peut être comprise en termes de feintise (cela, comme nous le verrons, est la position de Recanati), de telle sorte que ce qui serait nécessaire pour justifier une différence est la défense d'une différence - forte — entre la sorte de feintise qui est impliquée dans de simples échos et la sorte de feintise qui est impliquée dans l'ironie. Cela semble bien sûr être le but des remarques de Currie selon lesquelles la feintise impliquée n'est pas une feintise d'action (dont on peut penser qu'elle serait suffisante pour caractériser les échos), mais une feintise d'être. La façon exacte dont on doit comprendre cette proposition est cependant loin d'être claire. Les paragraphes suivants vont essayer de clarifier la contribution de la feintise à l'ironie, sa réductibilité éventuelle à la mention échoïque et en quoi, dans le cas spécifique de l'ironie, la notion d'une feintise d'être pourrait être impliquée. Commençons par les relations entre écho et feintise.

\section{Jusqu'à quel point la mention échoïque est-elle différente de la feintise ?}

La majeure partie de la discussion à venir sera formulée indifféremment en termes de feintise ou de simulation. Les deux termes ont été utilisés de façon plus ou moins interchangeable dans la littérature ${ }^{25}$, et je ne connais pas de

24. La sienne et celle de Sperber et Wilson.

25. Un terme additionnel est le terme de faire-semblant (cf. Walton, 1990). Imagination s'est récemment joint à cette liste de termes (cf. les articles dans le recueil édité par Nichols, 2006). 
discussion limpide de la relation entre l'un et l'autre qui montrerait une différence précise entre eux. De fait, la différence principale semble être que feintise peut être plus souvent utilisé dans le contexte de l'action (feintise d'action) et simulation dans le contexte des états mentaux (feintise d'être) ou dans celui du raisonnement contrefactuel. Mais cela semble être la totalité de la différence entre eux, et je suivrai la majeure partie de la littérature en les utilisant de façon indifférenciée.

Dans son livre sur l'oratio obliqua, Recanati $(2000,50)$ affirme que la simulation se produit dans tous les cas où nous «imaginons un monde différent du monde réel - quand nous prétendons que le monde est différent de ce qu'il est en fait ", que cette vision du monde comme différent de ce que nous savons qu'il est soit produite pour nous-mêmes (comme, on peut le supposer, c'est le cas dans les conditionnelles contrefactuelles) ou attribuée à quelqu'un d'autre (comme c'est le cas dans le discours ou la pensée rapportée, ou dans l'ironie). Comme Recanati le note, la proposition dont le contenu est simulé n'est pas sérieusement assertée.

Comme on le voit, dans cette optique, n'importe quel cas de mention échoïque, au sens de Sperber et Wilson, est un cas de feintise, ou de simulation dans le sens de Recanati. La question est de savoir si c'est un cas de feintise ou de simulation dans le sens de Currie. Cette dernière question revient à savoir si la vision de la feintise ou de la simulation de Currie est équivalente à celle de Recanati.

À première vue, Recanati ne semble pas distinguer entre feintise d'action et feintise d'être, alors que cela semble être la base de l'affirmation de Currie selon laquelle son analyse n'est pas réductible à celle de Sperber et Wilson. Une des raisons pour lesquelles Recanati ne fait pas cette distinction est probablement qu'il s'intéresse principalement à l'oratio obliqua où elle n'est pas très pertinente. Que l'on puisse lui donner une base solide n'en est pas moins une question centrale. Une distinction qui pourrait être pertinente a récemment été introduite : celle entre deux sortes de simulation, ou entre deux significations du mot simulation.

\section{Simulation profonde et simulation superficielle}

Bien que les termes profond et superficiel ne soient pas standard dans la littérature, je vais les utiliser par la suite pour éviter les variations terminologiques qu'on trouve dans les travaux qui font cette distinction. Un de ses premiers avocats est Goldman (2006 a et b) et la distinction a été principalement faite dans le domaine de l'imagination, et plus précisément dans le domaine des réactions à la fiction. Fondamentalement, Goldman (2006a) formule la dichotomie en distinguant deux sortes d'imagination : l'imagination suppositionnelle et l'imagination enactive (respectivement imagination-S et imagination-E). Selon lui :

L'imagination-S est typiquement formulée avec une proposition en "que", « $\mathrm{X}$ imagine que $p$ », où $p$ peut référer, sans restriction, à n'importe quelle 
sorte d'état de choses. Imaginer-S que $p$ est entretenir l'hypothèse que $p$, poser que $p$, supposer que $p$. À la différence de certaines formes d'imagination, l'imagination-S n'a pas d'aspect sensoriel, elle est purement conceptuelle. [...] L'imagination énactive réside dans le fait de créer ou de chercher à créer dans son propre esprit un état mental donné, ou au moins un facsimile approximatif d'un tel état, par la faculté d'imagination. Les exemples centraux d'imagination-E incluent des formes sensorielles d'imagination, où l'on crée, par l'imagination, des états quasi-perceptifs [perception-like]. [...] Tous les modes d'imagination-E, cependant, n'impliquent pas l'imagerie (Goldman 2006a, 42).

Goldman (2006a, 44) continue :

Il y a une différence substantielle entre la vision selon laquelle l'imagination-S couvre tous les usages importants de l'imagination dans la consommation de la fiction et la vision selon laquelle l'imagination-E [...] est essentielle à la consommation et à l'appréciation de la fiction.

Prima facie, il semblerait que l'analyse que propose Currie de l'ironie différerait de celle de Sperber et Wilson dans la mesure où elle lierait l'ironie avec l'imagination-E (ou la simulation-E) plutôt qu'avec l'imagination-S (ou la simulation-S). De fait, il semble clair que la façon dont Recanati utilise la notion de simulation ne nécessite rien d'aussi fort que la simulation-E: la simple simulation-S est tout à fait suffisante pour ce qu'il a à l'esprit.

Appelons simulation superficielle la simulation-S et simulation profonde la simulation-E. La base de l'affirmation de Currie semble être que son analyse en termes de feintise ne serait réductible à celle de Sperber et Wilson en termes d'écho que si la feintise impliquée dans l'ironie était une instance de simulation superficielle. Sa réfutation de la réductibilité est basée sur l'affirmation que la feintise impliquée dans l'ironie est, cependant, une instance de simulation profonde. Et, la simulation profonde étant largement irréductible à la simulation superficielle, l'analyse en termes de feintise est irréductible à l'analyse échoïque.

Cela soulève quelques autres questions :

- La feintise d'action est-elle vraiment une forme de simulation superficielle plutôt que de simulation profonde?

- La feintise d'être, impliquant la simulation profonde, est-elle vraiment impliquée dans l'ironie ? Et, si elle l'est, quel est son rôle exact?

Comme on l'a dit ci-dessus, il semble que Currie voit l'écho, en tant qu'il a à voir avec la feintise, comme une instance de feintise d'action, ou, dans la présente terminologie, de simulation superficielle, ou de simulation n'impliquant pas, dans les termes de Goldman, " le fait de créer ou de chercher à créer dans son propre esprit un état mental donné, ou au moins un facsimile approximatif d'un tel état mental, par la faculté d'imagination ». 
De fait, il y a des exemples où la mention échoïque est impliquée explicitement et où la simulation impliquée dans l'écho est superficielle (feintise d'action):

19. La nouvelle [de la mort de Péguy et de celle d'Alain Fournier] attriste Gallimard et le révolte: cette mort inattendue le confirme dans sa haine de la guerre, injuste pourvoyeuse de cimetière. Tuer des soldats, cela va de soi, c'est leur métier : mais des hommes comme Péguy, qui n'avait que quarante et un ans et tant à faire, à écrire, à éditer !... Et Alain Fournier! A vingt-huit ans il avait eu à peine le temps de commencer son œuvre! En ce début d'hiver 1914, Gallimard doit penser avec amertume et dérision à ce que Péguy, justement, disait à Fournier trois ans auparavant:

- Vous irez loin, Fournier. Vous vous rappellerez que c'est moi qui vous l'ai dit.

(Assouline, P., Gaston Gallimard: Un demi-siècle d'édition française, 79)

Mais ces exemples restent peu fréquents et semblent restreints à des cas où l'analyse de Sperber et Wilson s'appliquerait de façon forte: c.-à-d. avec une notion absolue de mention échoïque et aux échos d'énoncés effectivement produits. Incidemment, dans cet exemple, bien que la feintise superficielle soit réellement impliquée au travers de l'écho, il n'est pas clair que la simulation profonde soit impliquée dans le passage ironique lui-même.

Ainsi, il semble que l'affirmation de Currie selon laquelle la feintise impliquée dans l'écho est une feintise d'action, alors que la feintise impliquée dans l'ironie est une feintise d'être, n'est pas une critique valide de la théorie de Sperber et Wilson dans la mesure où, clairement, elle ne s'accorde pas avec la notion de l'écho qu'ils utilisent qui est (a) relative plutôt qu'absolue et (b) généralisée aux échos de pensées attribuées et pas seulement d'énoncés effectifs. Comme on le verra plus loin, cela semble impliquer chez l'agent des états mentaux cherchant à reproduire les états mentaux de la cible ${ }^{26}$. Dans cette mesure, bien que l'équation qu'opère Currie entre écho et feintise d'action puisse rendre compte de cas simples où l'énoncé ironique fait écho à un énoncé effectif comme, (12) par exemple, elle ne semble pas applicable à des cas plus complexes comme (13).

Ainsi, dans certains cas au moins, il semble que la feintise impliquée dans les énoncés ironiques échoïques est au moins autant de la feintise d'être (ou de la simulation profonde) que de la feintise d'action (simulation superficielle). Il n'est donc pas clair que cette ligne de défense contre la réduction de l'analyse en termes de feintise à l'analyse échoïque soit accessible à Currie. Bien évidemment, ce pourrait être à cause de la faiblesse même de l'analyse de Sperber et Wilson, telle qu'elle a été décrite ci-dessus (cf. $\$ 4$ ). Apparemment, l'analyse de Currie paraîtrait préférable parce qu'elle est plus forte: elle s'appuie sur

26. Bien que cela n'implique pas nécessairement des «formes sensorielles d'imagination » ou des «états quasi-perceptuels». 
un concept plus strict, c.-à-d. la feintise d'être ou la simulation profonde. Mais, et c'est la seconde question, est-ce plus qu'une apparence?

La feintise d'être, impliquant la simulation profonde, est-elle vraiment impliquée dans l'ironie ? Revenons-en à des exemples (en oubliant (12) qui passe décidément les limites acceptables de l'ennui...):

20. Un religieux de l'ordre de Saint Benoît, Ernold le Pingouin, effaça à lui seul quatre mille manuscrits grecs et latins, pour copier quatre mille fois l'évangile de Saint Jean. Ainsi furent détruits en grand nombre les chefs-d'œuvre de la poésie et de l'éloquence artistique. Les historiens sont unanimes à reconnaître que les couvents pingouins furent le refuge des lettres au moyen âge. (A. France, L'île des pingouins, 107-108).

21. La belle princesse recommanda le secret à ses dames sur ce qu'elles avaient vu. Elles le promirent toutes, et en effet le gardèrent un jour entier (Voltaire, Le Taureau blanc, 242).

22. Le plaisant de l'éducation actuelle, c'est qu'on n'apprend rien aux jeunes filles qu'elles ne doivent oublier bien vite dès qu'elles seront mariées (Stendhal, De l'amour, 244).

23. Il est du véritable amour comme de l'apparition des esprits : tout le monde en parle, mais peu de gens en ont vu (La Rochefoucauld, Maximes, 76.)

24. On a remarqué qu'à Constance il y avait eu sept cent dix-huit filles pour le service du concile qui fit brûler si dévotement Jean Hus et Jérôme de Prague (Voltaire, L’homme aux 40 écus, 164).

25. Rien n'était si beau, si leste, si brillant, si bien ordonné que les deux armées. [...]. Les canons renversèrent d'abord à peu près six mille hommes de chaque côté; ensuite la mouqueterie ôta du meilleur des mondes environ neuf à dix mille coquins qui en infectaient la surface. La baïonnette fut aussi la raison suffisante de la mort de quelques milliers d'hommes. Le tout pouvait bien se monter à une trentaine de mille âmes. Candide, qui tremblait comme un philosophe, se cacha du mieux qu'il put pendant cette boucherie héroïque.

Enfin, tandis que les deux rois faisaient chanter des Te Deum chacun dans son camp, il prit le parti d'aller raisonner ailleurs des effets et des causes (Voltaire, Candide, 14).

26. Je vénère les antiquités pingouines et j'ai le culte des primitifs. Ils sont délicieux. Je ne dis pas qu'ils se ressemblent tous; ce ne serait point vrai; mais ils ont des caractères communs qu'on retrouve dans toutes les écoles; je veux dire des formules dont ils ne sortent point, et quelque chose d'achevé, car ce qu'ils savent ils le savent bien. On peut heureusement se faire une idée des primitifs pingouins par les primitifs italiens, flamands, allemands et par les primitifs français qui sont supérieurs à tous les autres; comme le dit M. Gruyter, ils ont plus de logique, la logique étant une qualité spécialement française (France, A. L'île des pingouins, 113-114). 
27. Pourvu qu'on ne plaisantât ni de Dieu, ni des prêtres, ni du roi, ni des gens en place, ni des artistes protégés par la cour, ni de tout ce qui est établi; pourvu qu'on ne dît du bien ni de Béranger, ni des journaux de l'opposition, ni de Voltaire, ni de Rousseau, ni de tout ce qui se permet un peu de franc-parler; pourvu surtout qu'on ne parlât jamais politique, on pouvait librement raisonner de tout (Stendhal, Le Rouge et le Noir, 576).

Il y a deux points sur lesquels on peut examiner ces exemples : d'abord, impliquent-ils, ou non, un écho ? ensuite, impliquent-ils une simulation profonde ? Nous les examinerons d'abord pour la première question, en revenant à la seconde lorsque la notion de simulation profonde aura été davantage précisée. Prima facie, (20), (26) et (27) impliquent de façon évidente un écho. La situation est moins claire pour (21) et (24), bien que l'on puisse défendre la notion d'un écho partiel : selon cette analyse, [elles] le gardèrent un jour entier en (21) et si dévotement en (24) pourraient être des échos partiels, le reste de l'énoncé servant de contexte ${ }^{27}$. Une analyse échoïque serait très difficile à défendre pour (22), (23) ${ }^{28}$ et, plus encore, pour des raisons déjà exposées plus haut, pour (25). Comme on l'a vu, en suivant la première direction de la description de l'imagination-E donnée par Goldman (simulation profonde), en termes de création d'un état mental donné, il semble difficile de dire que les échos sont de simples instances de la simulation superficielle (imagination-S) : par exemple, aussi longtemps que l'on admet que, dans un énoncé ironique, ce ne sont pas seulement des énoncés effectifs antérieurs, mais des pensées auxquels on peut faire écho, il est clair que l'on doit accéder ${ }^{29}$, d'une façon ou d'une autre, à de telles pensées et comment pourrait-on y accéder sans les entretenir ? Cependant, la description que propose Goldman de l'imagination-E (ou simulation profonde ou feintise d'être) s'appuie aussi sur une seconde caractéristique : " des exemples centraux de l'imagination-E incluent des formes sensorielles d'imagination, où on crée, par l'imagination, des états quasi-perceptifs ». Les exemples qui peuvent être analysés en termes échoïques impliquent-ils cette seconde caractéristique ? Clairement, ni (20), (26) ou (27) ne le font. Mais ce sont aussi les cas où l'on peut le plus légitimement supposer qu'il y a un énoncé effectif dont l'énoncé ironique est l'écho. Qu'en est-il de cas plus éloignés ? Par exemple des cas où l'écho pourrait être seulement partiel (c.-à-d. où seulement une partie de l'énoncé ironique peut être considéré comme écho),

27. On pourrait probablement défendre une telle analyse en adoptant certaines propositions de Recanati (2000), mais je n'argumenterai pas ici pour cette analyse.

28. Bien que, comme on l'a vu plus haut (cf. $\$ 1$ ), le statut ironique de (23) soit discutable.

29. Il faut reconnaitre que l'on peut supposer que l'accès aux pensées pourrait se faire par un processus purement "théorique » et, étant donné leur insistance (présente) sur le caractère modulaire et inné de la théorie de l'esprit, c'est probablement la position qu'adopteraient Sperber et Wilson. Mais rien dans leur analyse de l'ironie en termes de mention échoïque ne repose sur cette insistance. En elle-même, leur analyse est parfaitement compatible avec une analyse simulationniste de la théorie de l'esprit. Dans une telle analyse, étant donné les variétés alternatives superficielle et profonde et étant donné la première caractérisation de Goldman, la simulation profonde semble s'imposer. J'y reviendrai. 
comme en (21) et (24) ? Pourrions-nous dire dans de tels cas qu'ils ne satisfont pas seulement la première caractéristique évoquée par Goldman (susciter un état mental donné dans son propre esprit) pour la simulation profonde (imagination-E), mais que la seconde (l'accompagnement de sensations) l'est aussi ? Cela semble très difficile: pourquoi de telles simulations devraient-elles donner lieu à des états quasi-perceptuels ou sensoriels ?

Qu'en est-il des cas où une analyse échoïque paraît difficile à maintenir ? Si l'on ignore le cas douteux de (23), cela laisse (22) et (25). Or (22) est le mot d'esprit de Stendhal sur l'éducation des filles : il est difficile de voir en quoi il pourrait impliquer quelque chose comme des états " quasi-perceptuels». Si vous ne trouvez pas que (22) est un exemple acceptable d'ironie, ou, en d'autres mots, si vous pensez que c'est un cas limite, que faut-il dire de (25) qui est considéré depuis longtemps comme un exemple standard ? Comme on l'a vu plus haut (cf. $\$ 4$ ), c'est la description de deux événements. Mis à part le fait que ces événements sont intentionnels - et indiquent donc l'état mental, l'intention, de leurs agents - il est difficile de voir pourquoi un état sensoriel, quel qu'il soit, imaginaire ou non, devrait être impliqué... De fait, il est difficile de voir où, exactement, la feintise intervient ${ }^{30}$. Autrement dit, (25) n'est pas moins fatal pour l'analyse de l'ironie en termes de feintise qu'il ne l'était pour l'analyse échoïque ou pour l'analyse antiphrastique. Et les cas dans lesquels l'analyse en termes de feintise s'applique le plus fortement qui, incidemment, coïncident (cf. ci-dessous) avec les cas où l'analyse échoïque semble largement confirmée - ne sont pas des cas où la variété profonde de feintise est pleinement active. De fait, il semble que la feintise opérationnelle dans les cas d'ironie qui semblent la vérifier fortement s'appuie principalement sur des bases conceptuelles, plutôt que sensorielles ${ }^{31}$. Cela, soit dit en passant, s'accorde très bien avec l'idée de Currie selon laquelle l'ironie a à voir avec une évaluation de raisonnabilité.

Tout cela laisse cependant sans réponse la question subsidiaire posée cidessus : si la feintise d'être, impliquant une variété profonde (bien que possiblement non sensorielle) de simulation, est présente dans l'ironie, où, exactement, est-elle impliquée?

\section{Ironie et simulation mentale}

Selon moi, la simulation profonde est effectivement impliquée dans l'ironie, mais seulement de façon contingente - principalement au travers de la mention échoïque - dans les énoncés ironiques eux-mêmes. Elle est impliquée dans

30. Mis à part le fait, bien sûr, que cet exemple est tiré d'une fiction... si l'on accepte une analyse en termes de feintise (profonde) de la fiction... Mais cela est une autre question.

31. Cela ne contredit bien évidemment pas l'analyse en termes de feintise: comme Goldman (2006b) le remarque justement, aucune théorie de la feintise ne peut se passer de concept. Il n'y a là aucun problème, mais cela ne justifie certainement pas, dans le cas spécifique de l'ironie, une analyse en termes de feintise profonde. 
l'ironie dans la mesure où l'ironie met en lumière des erreurs de raisonnabilité (c'est une approximation qui sera précisée ci-dessous) et que ceci suppose que le locuteur de l'énoncé ironique a reconstitué une instance de raisonnement qu'il évalue, à tort ou à raison, comme déraisonnable. Cela n'est pas cependant - ou n'est pas nécessairement - ce qui se produit dans la production même de l'énoncé ironique. Plutôt, c'est un processus qui sous-tend la production de l'énoncé ironique. En d'autres termes, c'est le processus par lequel, par exemple, en (21), Voltaire se moque de façon implicite des dames d'honneur dans la mesure où, si l'on suppose que leur promesse est tenue, elle est tenue seulement dans la mesure où elles gardent le secret, non pas pour le reste de leur vie, mais durant une unique journée. C'est, de fait, par la confrontation entre les deux, remplir sa promesse en gardant le secret tout le reste de sa vie et remplir sa promesse en gardant le secret un seul jour, que l'ironie est suscitée. Autrement dit, Voltaire simule ce que serait garder un secret selon les dames d'honneur, évalue le caractère raisonnable de cette vision des choses, et rend accessible le contraste à ses lecteurs, en le mettant en lumière via le connecteur en effet. Cette simulation peut être plus ou moins directement accessible dans l'énoncé ironique lui-même (et, dans de tels cas, on peut parier que l'analyse en termes de mention échoïque s'applique aussi), comme en (28):

28. Après le tremblement de terre qui avait détruit les trois quarts de Lisbonne, les sages du pays n'avaient pas trouvé un moyen plus efficace pour prévenir une ruine totale que de donner au peuple un bel autodafé; il fut décidé par l'université de Coïmbre que le spectacle de quelques personnes brûlées à petit feu, en grande cérémonie, était un secret infaillible pour empêcher la terre de trembler (Voltaire, Candide ou l'optimiste, 23)

Mais c'est l'étendue maximale de la simulation profonde dans la production ironique. Il devrait être évident que la simulation profonde est aussi impliquée dans l'interprétation ironique, comme le montre l'exemple (25) où l'ironie dépend directement de la reconstruction dans son propre esprit de la pensée (commune) des deux rois, c.-à-d., J'ai gagné la bataille. Cela nous conduit directement au point final dont parle Currie dans son article : l'impossibilité, selon lui, d'une ironie auctoriale dans un roman.

\section{Un argument massue : l'ironie auctoriale}

L'ironie auctoriale est ce qui se produit quand un personnage, dans une fiction, dit (sérieusement) quelque chose qui est interprété par le lecteur comme absurde ou ridicule d'une façon ou d'une autre. Elle doit être distinguée de ce qui se produit quand un personnage fait une remarque ironique ciblant un autre personnage. Dans le premier cas, l'énoncé n'est pas ironique dans la fiction alors que dans le second il l'est. Ces deux cas sont illustrés simultanément dans l'exemple suivant tiré de Tartuffe $(\mathrm{I}, 5)$ : 


\section{6 - Philosophiques / Printemps 2008}

\section{ORGON}

$[\ldots]$

Oui, je deviens tout autre avec son entretien;

Il m'enseigne à n'avoir d'affection pour rien,

De toutes amitiés il détache mon âme;

Et je verrais mourir frère, enfants, mère et femme,

Que je m'en soucierais autant que de cela.

\section{CLÉANTE}

Les sentiments humains, mon frère, que voilà !

Dans cet exemple, la remarque finale de Cléante est un énoncé ironique qui prend pour cible Orgon : c'est un exemple typique d'ironie de personnage, où l'énoncé est ironique dans la fiction. Le discours d'Orgon, par contraste, n'est certainement pas de l'ironie de personnage: Orgon fait l'éloge de Tartuffe qui lui a appris, au nom du Christ (Tartuffe fait semblant d'être un bon chrétien, tout en pique-assiettant chez Orgon), à ignorer les sentiments mondains que sont l'amour pour sa famille et l'amitié. Cependant, du point de vue du spectateur, hors de la fiction, il n'y a pas de doute que ce discours est ironique et que Molière prend pour cible la sottise d'Orgon, conduisant le public à prendre son parti plutôt que celui du personnage ${ }^{32}$. Currie propose un exemple d'ironie auctoriale du même type dans son article, pris cependant, non pas dans le théâtre, mais dans le cinéma. Cependant, il affirme dans l'analyse qu'il en donne qu'il ne peut y avoir d'ironie auctoriale dans la fiction littéraire :

[Les personnages] sont représentés comme occupant des perspectives absurdement défectueuses, et c'est en partie ce qui rend leurs remarques drôles. Dans cette mesure, leurs remarques sont drôles parce qu'elles ne sont pas ironiques. Mais elles sont aussi drôles parce que les mots sont prononcés par de vrais acteurs (cette technique ne marche pas dans la fiction littéraire) avec lesquels nous partageons la conscience des potentialités ironiques de la remarque ${ }^{33}$ (Currie 2006, 130).

Je pense que cela est absolument faux et je vais consacrer une bonne partie du reste de cet article à le montrer.

Pour l'instant, cependant, la question la plus intéressante est de savoir pourquoi Currie devrait refuser l'ironie auctoriale dans la fiction littéraire. Revenons-en rapidement à son analyse de l'ironie :

- Caractéristique centrale : l'ironie implique de la feintise ${ }^{34}$;

- Première caractéristique additionnelle : l'ironie a une cible;

32. De fait, on peut considérer que l'ironie de personnage dans le commentaire de Cléante est clairement intentionnée (par Molière) pour rendre l'ironie auctoriale présente dans le discours d'Orgon évidente pour le public, en mettant en lumière son caractère déraisonnable.

33. Les italiques sont de moi.

34. J'ignore la distinction entre feintise d'action et feintise d'être parce qu'elle n'est pas pertinente ici, comme nous allons bientôt le voir. 
- Seconde caractéristique additionnelle: l'ironie exprime une attitude - le plus souvent, mais pas toujours — négative à l'encontre de la raisonnabilité de la cible.

Les deux caractéristiques additionnelles sont indiscutables. Mais ce qui est en débat dans la discussion sur l'ironie auctoriale est, de façon peu surprenante, la caractéristique centrale, c'est-à-dire la feintise (quelle que soit sa nature). Pourquoi l'existence de l'ironie auctoriale devrait-elle faire obstacle à l'analyse en termes de feintise de l'ironie?

Si l'on en revient à (29), on y trouve tout à la fois de l'ironie de personnage (la répartie de Cléante après le grotesque discours d'Orgon) et de l'ironie auctoriale (dans le théâtre cependant, ce qui, d'après Currie, lui ôterait toute incidence) dans la tirade ridicule d'Orgon. Essayons de produire une analyse de ces deux types d'ironie selon les termes de l'analyse que propose Currie tout à la fois de la fiction (dramatique ou non) et de l'ironie. Selon Currie (je n'entrerai pas dans les détails, qui, d'ailleurs, ne sont pas pertinents ici), la fiction implique de la feintise. C'est aussi le cas de l'ironie. Commençons par examiner l'ironie (de personnage) chez Cléante :

$\star \quad$ Il est fictionnel que $\left[1^{\circ}\right.$ niveau de feintise $]$ Cléante dit que $\left[2^{\circ}\right.$ niveau de feintise] il admire les sentiments humains d'Orgon.

Il semblerait que dans ce cas l'analyse que propose Currie de l'ironie soit validée: il y a, effectivement, deux niveaux de feintise, le premier lié à la fiction, le second lié à l'ironie de personnage. Autrement dit, comme on l'a vu ci-dessus, il est fictionnel (= vrai dans la fiction) que Cléante soit ironique à l'égard d'Orgon. Mais, comme on l'a dit ci-dessus, ce ne peut pas être le cas pour l'ironie auctoriale dans des exemples comme le discours grotesque d'Orgon, parce que le personnage n'est pas ironique. Ainsi, ce ne peut être le cas que : il est fictionnel (= vrai dans la fiction) qu'Orgon soit ironique; ce n'est pas davantage le cas qu'il est fictionnel (= vrai dans la fiction) que Molière (ou un narrateur commode ${ }^{35}$ ) soit ironique, parce que, clairement, ce n'est pas vrai dans la fiction. Ainsi il semble que l'ironie auctoriale soulève un énorme problème pour l'analyse de l'ironie en termes de feintise.

Examinons rapidement ce que Currie a à dire de l'ironie auctoriale dans la fiction dramatique ${ }^{36}$. Selon lui :

[de tels exemples sont] drôles parce qu'ils ne sont pas ironiques [et, effectivement, ils ne le sont pas dans la fiction]. Mais ils sont aussi drôles parce que les mots sont prononcés par de vrais acteurs (cette technique ne marche pas dans la fiction littéraire) avec lesquels nous partageons la conscience des potentialités ironiques de la remarque (cf. la citation complète ci-dessus).

35. Pour une discussion approfondie des difficultés que soulève l'ironie auctoriale dans le théâtre pour la narratologie, cf. Reboul 1984).

36. Pour la commodité de l'exposé, j'utiliserai fiction dramatique pour référer à tous les cas de fiction dans lesquels des acteurs sont impliqués. 
Cela semble quelque peu obscur : le discours d'Orgon n'est pas ironique: de fait, c'est parce qu'il ne l'est pas qu'il est drôle (mais, dès lors, qu'y a-t-il exactement de drôle dans ce discours ?) ; il est drôle parce que — au moins lorsqu'on le voit sur scène - l'acteur partage avec nous « la conscience des potentialités ironiques de la remarque ». Mais qu'est-ce qu'une potentialité ironique? En fait, comment pouvons-nous avoir des potentialités ironiques quand il n'y a pas de feintise si la caractéristique centrale de l'ironie est la feintise et s'il n'y a pas de feintise (additionnelle, spécifique à l'ironie) dans de tels cas ?

Une réponse possible consisterait à utiliser les caractéristiques secondaires de l'ironie : la présence d'une cible et la détection d'un caractère déraisonnable. Mais, en l'absence de feintise, comment la cible peut-elle être détectée et comment la détection du caractère déraisonnable se fait-elle ? Et pourquoi - si l'existence d'une cible est la base du caractère humoristique de l'ironie - l'existence même d'une déraison serait-elle suffisante, en elle-même, en l'absence d'ironie - impliquée, selon Currie, par l'absence de feintise (de second niveau, spécifique à l'ironie) - pour déclencher l'hilarité ? De fait, quand la déraison, par elle-même, a-t-elle déclenché l'hilarité ? Il serait bon que ce soit le cas, mais ce n'est clairement pas ce qui se produit, pas plus d'ailleurs que la déraison n'est immédiatement reconnue comme telle quand elle se produit. Un bon exemple de ce regrettable aveuglement est que «en dépit des preuves photographiques, en 1933, le périodique Die Sonne affirma à ses lecteurs qu'Hitler était un blond aux yeux bleus » (Mosse 2006, 132). Le simple fait que ce périodique n'ait pas immédiatement perdu l'ensemble de son lectorat indique avec éclat l'étendue de la tolérance humaine pour la sottise ${ }^{37}$. Ainsi la notion de "potentialités ironiques » reste mystérieuse.

Pourrions-nous passer par la reconnaissance de la déraison par les acteurs pour nous aider à faire sens de ce qui rend drôle le discours d'Orgon ? Pourrions-nous, d'une façon ou d'une autre, construire une analyse semblable à celle de l'ironie de personnage pour expliquer le comique de ce discours? Il faudrait qu'elle dise quelque chose comme :

$\star$ Il est fictionnel $\left(=\right.$ vrai dans la fiction $\left.{ }^{38}\right)$ que $\left[1^{\circ}\right.$ nivean de feintise l'acteur voit que ( $2^{\circ}$ niveau de feintise Orgon dit quelque chose de déraisonnable)]

Il est clair que cela ne va pas. L'acteur n'est pas dans la portée du premier niveau de feintise (même si la fiction porte sur la performance elle-même): plutôt, c'est Orgon qui est dans la portée de ce premier niveau. Donc, de nouveau, nous n'avons pas deux niveaux de feintise, comme nous le devrions si l'analyse de Currie était correcte. Arrêtons-nous un instant: Currie dit que ce n'est pas de l'ironie (mais seulement — et mystérieusement — des potentialités ironiques). Ainsi, il est parfaitement compatible avec son analyse

37. De fait, comme la reine rouge de Lewis Carroll, nous semblons prêts à croire au moins six choses impossibles avant le petit déjeuner, comme le montre tristement l'histoire électorale dans les pays démocratiques.

38. Où la fiction est le fait de jouer la pièce. 
qu'il n'y ait pas deux niveaux de feintise ici. Mais nous n'avons que la parole de Currie pour considérer qu'il n'y a pas là ironie.

De fait, l'énigme s'épaissit avec l'affirmation suivante de Currie : nous pouvons trouver le discours d'Orgon drôle parce que nous partageons avec l'acteur la perception de ses potentialités ironiques. Cela ne fonctionnerait pas dans la fiction littéraire parce qu'il n'y a pas, dans la fiction littéraire, d'acteur avec qui partager cette perception. Cela est troublant: Currie veut-il dire que rien ne peut être vu comme drôle dans la fiction littéraire? Mais il y a autant de romans comiques que de comédies. Et pourquoi une conscience de la déraison d'un personnage, commune avec l'acteur, devrait-elle être la racine de notre rire dans la fiction dramatique plutôt qu'une conscience commune... avec l'auteur ? Ou devrions-nous penser que c'est l'acteur qui a mis la déraison dans la fiction dramatique et retirer à l'auteur toute responsabilité dans les actions du personnage?

De fait, avons-nous une raison quelconque de ne pas penser qu'il y a de l'ironie dans le discours d'Orgon mis à part le fait que reconnaître qu'il y en a contredirait l'analyse de Currie en termes de feintise? Et, si nous n'en avons pas - en tenant compte de l'aveu partiel de Currie lorsqu'il parle de "potentialités ironiques » -, le refus de l'ironie auctoriale, dans la fiction dramatique ou littéraire, est-elle autre chose qu'une pétition de principe?

\section{Y a-t-il de l'ironie auctoriale dans la fiction littéraire ?}

Un des exemples les plus riches d'ironie auctoriale du type de celle dont Currie affirme qu'elle n'existe pas se trouve dans la littérature anglaise, plus précisément dans Barry Lindon. C'est l'histoire d'une canaille, un jeune Irlandais d'extraction douteuse, tout à la fois tricheur aux cartes, escroc occasionnel et Don Juan, qui atteint les sommets de sa fortune lorsqu'il épouse une riche et aristocratique veuve anglaise qui a un fils de son premier mariage. Il dépense sa fortune et les maltraite, elle et son fils, avant d'être jeté dehors par sa belle-famille. L'ensemble du roman est une autobiographie (fictive), supposément écrite par Barry Lindon lui-même. Clairement, si quelque chose d'ironique (hors de l'ironie de personnage) peut être trouvé dans Barry Lindon, ce doit être un exemple d'ironie auctoriale, se produisant - contrairement aux prédictions de Currie - dans une fiction littéraire. Je pense que quiconque lit ce livre ne peut manquer de trouver de tels exemples. De fait, le premier apparaît dans la première phrase:

30. Since the days of Adam, there has been hardly a mischief done in this world but a woman has been at the bottom of it (Thackeray, W. M., Barry Lindon, 13).

[Depuis Adam, il n'y a pratiquement pas eu de mal fait en ce monde sans qu'une femme ne l'ait provoqué.]

Dans ce cas spécifique, on pourrait dire que l'ironie peut seulement apparaître par la suite lorsque le contexte (c.-à-d. les exploits douteux de Barry 
Lindon en ce qui concerne les femmes) est devenu évident. Cela ne devrait cependant pas faire croire que (30) n'est pas ironique, ou que ce n'est pas de l'ironie auctoriale. Mais il y a des exemples encore plus clairs:

31. My faults are many, I confess, and as a domestic character, I can't boast of any particular regularity, or temper; but Lady Lindon and I didn't quarrel more than fashionable people do, and, at first, we always used to make it up pretty well. I am a man full of errors, certainly, but not the devil that these odious backbiters of Tiptoff represented me to be. For the first three years I never struck my wife but when I was in liquor. When I flung the carving-knife at Bullingdon I was drunk, as everybody present can testify; but as for having any systematic scheme against the poor lad, I can declare solemnly that, beyond merely hating him (and one's inclinations are not in one's power), I am guilty of no evil towards him (Thackeray, W. M., Barry Lindon, 267).

[J'ai de nombreux défauts, je le confesse, et comme personnage domestique, je ne peux me vanter d'avoir l'humeur égale ou agréable; mais Lady Lindon et moi ne nous sommes pas querellés davantage que ne le font les gens à la mode et, au début, nous nous réconciliions facilement. Je suis plein d'erreurs, sans aucun doute, mais je ne suis pas le démon que ces odieux médisants de Tiptoff ont dit. Pendant les trois premières années, je n'ai jamais frappé ma femme sans être saoul. Quand j'ai lancé le coupeau à découper sur Bullingdon j'étais ivre, comme tous les gens présents peuvent en témoigner; mais loin d'avoir un plan systématique contre le pauvre garçon, je peux déclarer solennellement que, mis à part le fait que je le déteste (et on ne contrôle pas ses sentiments), je ne suis coupable de rien de mal en ce qui le concerne.]

32. In fact, I can't conceive how such a shabby rogue as that could ever have had the audacity to propose for her: his birth was certainly good; but what other qualifications had he? (Thackeray, W. M., Barry Lindon, 159).

[En fait, je n'arrive pas à imaginer comment une aussi misérable canaille a jamais pu avoir l'audace de lui demander sa main : il était certainement de bonne naissance; mais quelles autres qualifications avait-il ?]

Je suppose que (31) peut se passer de commentaire. En (32), Barry Lindon, de naissance douteuse et sans fortune, attaque un de ses rivaux pour la main de sa (future à ce moment) épouse, sans apparemment se rendre compte que de pires reproches pourraient lui avoir été faits à lui.

Au cas, cependant, où le lecteur penserait que cet exemple est exceptionnel et que Barry Lindon est un cas unique, voici quelques autres exemples:

33. Comme prenves les pièces fausses, en général, valent mieux que les vraies, d'abord parce qu'elles ont été faites exprès, pour les besoins de la cause, sur commande et sur mesure, et qu'elles sont enfin exactes et justes. Elles sont préférables aussi parce qu'elles transportent les esprits dans un monde idéal et les détournent de la réalité qui, en ce monde, hélas! n'est jamais sans mélange... (France, A., L'Île des pingouins, 209). 
34. - Point du tout, répliqua [Pangloss]; c'était une chose indispensable dans le meilleur des mondes, un ingrédient nécessaire; car si Colomb n'avait pas attrapé, dans une île de l'Amérique, cette maladie qui empoisonne la source de la génération, qui souvent même empêche la génération, et qui est évidemment l'opposé du grand but de la nature, nous n'aurions ni le chocolat ni la cochenille; il faut encore observer que jusqu'aujourd'hui, dans notre continent, cette maladie nous est particulière, comme la controverse (Voltaire, Candide ou l'Optimiste, 18).

En (33), le personnage qui parle est le ministre de la Justice ${ }^{39}$, persuadant ses subordonnés de fabriquer les preuves qui conduiront à la condamnation d'un innocent. En (34), Pangloss (une parodie fictive d'un disciple de Leibniz) défend envers et contre tout une vision selon laquelle ce monde est le meilleur des mondes, parce que si Colomb n’avait pas attrapé la syphilis, nous n’aurions ni le chocolat ni la cochenille...

Il y a donc clairement des exemples d'ironie auctoriale dans la fiction littéraire. Dans l'ironie auctoriale, il n'est pas fictionnellement vrai que l'énoncé est ironique. Il n'en est pas moins ironique en ceci que l'auteur rend évident pour le lecteur que quelque chose qui est fait, dit ou pensé par un personnage est déraisonnable. Dans ce cas, clairement, le côté humoristique des énoncés n'est pas dû au fait que des acteurs les trouvent drôles. Plutôt, ce que fait l'auteur est d'attirer son lecteur dans une collusion ironique contre le personnage, ou, plus généralement, contre le type de raisonnement que le personnage instancie. Cela nous conduira, au bout du compte, à proposer une analyse (positive) de l'ironie qui validera en partie toutes les analyses antérieures, y compris celles en termes de mention échoïque et de feintise.

\section{Un usage ironique de l'ironie auctoriale}

La meilleure preuve, peut-être, de l'existence de l'ironie auctoriale est le fait même que certains auteurs l'utilisent pour la nier. Le type de phénomène auquel je pense apparaît le plus souvent chez Stendhal ${ }^{40}$ :

35. Et d'ailleurs, se disait-il, si les Français ont du plaisir à être menés monarchiquement et tambour battant pourquoi les déranger ? La majorité aime apparemment cet ensemble doucereux d'hypocrisie et de mensonge qu'on appelle gouvernement représentatif** * C’est un républicain qui parle (Stendhal, Lucien Leuwen, 60).

39. Ce passage du roman (satirique) d'Anatole France est une version (fictive, mais fidèle) de l'affaire Dreyfus. Le ministre défend (dans la fiction) ce qui a eu lieu dans la réalité, à savoir la fabrication de "preuves».

40. Stendhal n'était cependant pas le premier, et le même type de phénomène - y compris les fausses notes de bas de page - se retrouve dans les contes de Voltaire (par exemple, dans Les Lettres d'Amabed, 185-186). 
36. Au moment où nous le prenons, cet ennemi des cigares [LL] ne pensait guère plus à la république, qui tarde trop à venir*.

* Dans l'opinion du héros, qui est fou et qui se corrigera (Stendhal, Lucien Leuwen, 60).

37. Mais quant à moi, pensait-il, je serais bien dupe de vivre encore deux mois dans ce séjour dégoûtant, en butte à tout ce que la faction patricienne peut inventer d'infâme et d'humiliant", et ayant pour unique consolation les imprécations de cette folle...

*C'est un jacobin qui parle (Stendhal, Le Rouge et le Noir, 787).

Que fait Stendhal dans ces exemples ? De façon peu surprenante, il fait un usage sophistiqué d'une (fausse) ironie auctoriale. En d'autres termes, il feint (dans les «notes de bas de page ») de penser que la pensée du personnage est déraisonnable. Pour ce faire, il fait quelque chose de proche de ce que fait Molière dans l'exemple (29) quand il utilise l'ironie de personnage (le commentaire de Cléante sur le discours d'Orgon) pour attirer l'attention du public sur le côté grotesque du discours de celui-ci. Dans cet exemple, Molière se dissocie clairement des croyances d'Orgon au travers du commentaire de Cléante. Stendhal, quant à lui, prétend se dissocier des croyances de son personnage en adoptant, dans ses notes de bas de page, l'opinion admise de son époque (grossièrement la restauration de la monarchie française durant la première moitié du XIX ${ }^{\mathrm{e}}$ siècle), présentant ainsi l'énoncé qui rapporte les croyances de ce personnage comme un cas d'ironie auctoriale. Cependant, c'est simplement un cas de feintise : de fait, Stendhal est d'accord avec le personnage. En d'autres termes, Stendhal ne se moque pas de son personnage : il se moque d'un lectorat hypothétique qui considérerait l'opinion du personnage comme déraisonnable. Mais le simple fait qu'il peut utiliser un tel mécanisme est un argument en faveur de l'existence de l'ironie auctoriale.

\section{Une synthèse}

À partir des remarques ci-dessus, il semble que ni l'analyse en termes de mention échoïque (Sperber et Wilson, 1981, 1995) ni l'analyse en termes de feintise (Currie, 2006) ne soient entièrement satisfaisantes. Il y a des cas avérés d'ironie où aucun écho n'est impliqué. Qui plus est, l'analyse échoïque peut seulement s'étendre au point où la conduisent Sperber et Wilson par un affaiblissement significatif de son contenu. En ce qui concerne l'analyse en termes de feintise, il n'est pas clair qu'elle s'en sorte mieux : de fait les cas les plus clairs de feintise semblent être des cas où l'analyse échoïque s'appliquerait tout aussi bien. Cela n'est pas un hasard : il y a clairement un lien étroit entre le fait que quelque chose est un écho et le fait que quelque chose est une feintise. Bien que l'analyse en termes de feintise prétende être plus forte que l'analyse échoïque parce qu'elle s'appuierait sur une sorte plus forte de feintise, cela ne semble pas confirmé par les faits. Et sa seule façon de traiter certains exemples d'ironie, c.-à-d. l'ironie auctoriale dans ou hors de la fiction dramatique, est 
de nier leur caractère ironique, apparemment parce qu'ils ne justifient pas l'analyse en termes de feintise, faisant ainsi une pétition de principe.

Cela, cependant, ne signifie pas que l'ironie n'est jamais échoïque ni qu'elle n'est jamais une feintise. De fait, un grand nombre d'exemples vérifient ces deux analyses contemporaines de l'ironie. Mais un grand nombre d'exemples vérifient aussi une analyse en termes de contre-vérité. Rien de tout cela, de façon peu surprenante, n'est suffisant pour une analyse " unitaire " de la fiction. Je vais maintenant proposer ma propre version d'une telle analyse.

\section{Conclusion : I'ironie comme montrer plutôt que comme dire}

Ma vision personnelle est que l'ironie est d'abord et avant toute chose un phénomène interprétatif. En d'autres termes (et cela nous permet de faire sens de la façon dont Currie parle de potentialités ironiques - une chose que sa propre analyse ne fait pas), nous avons tendance à prendre un énoncé donné ${ }^{41}$ comme ironique quand nous le voyons comme nous rendant évident intentionnellement un raisonnement ou un type de raisonnement qui nous frappe (et, du moins nous le supposons, à tort ou à raison, qui frappe le producteur de l'ironie) comme déraisonnable. Autrement dit, nous reconnaissons un énoncé comme ironique parce que nous pensons que le locuteur avait l'intention de produire en nous un effet (ironique) par la reconnaissance de cette intention. C'est, bien évidemment, un cas de signification NN (d'où la nécessité d'une approche gricéenne), mais ce n'est pas un cas d'implicature, entre autres parce que l'ironie est un cas prototypique de montrer plutôt que de dire. Dans l'ironie, on a la confrontation (explicite ou implicite) entre deux visions, l'une qui est adoptée à la fois par le locuteur et par l'interlocuteur, l'autre qui est attribuée à la cible. La différence entre ces deux visions est la racine de l'humour dans l'ironie. Dans des exemples comme (35) à (37), Stendhal fait un usage subtil de ce mécanisme, en prétendant prendre le parti d'un public putatif contre le personnage, alors qu'il prend celui du personnage et (il l'espère) d'une partie de son lectorat contre ce public hypothétique (identifié au gouvernement de l'époque et à ses thuriféraires).

Cette analyse en termes de "montrer", on le remarquera, explique pourquoi tout à la fois l'écho et la feintise (et la contre-vérité !) sont fréquents dans l'ironie, mais elle fait aussi sens d'exemples, comme (25), où il n'y a clairement ni écho ni feintise. De fait, des exemples comme les suivants font davantage sens dans une telle analyse que dans une analyse échoïque ou que dans une analyse en termes de feintise:

38. Quand on arrête maintenant des médecins juifs, des collègues aryens sont présents pour les identifier afin qu'il n'y ait pas de malentendu. Ils ont aussi

41. Ou un acte non verbal. 
la mission stricte, quand quelqu'un est maltraité, sans égard pour la personne, de délivrer une expertise disant que rien n'est arrivé, que le patient est encore résistant ou qu'il n'y a rien de suspect sur le cadavre, ce par quoi l'humour retrouve parfois ses droits (K. Kraus, Troisième nuit de Walpurgis, 441).

39. La pensée national-socialiste fascine par sa capacité à faire croire que celui qui dit une fois la vérité est crédible pour tous les mensonges et à présenter le vol, qu'exceptionnellement il n'a pas commis, comme l'alibi pour des milliers de meurtres. Qui sait? Peut-être que même un cas avéré ne serait pas un handicap à sa confirmation (K. Kraus, Troisième nuit de Walpurgis, 388).

À première vue, on pourrait penser que Kraus se contente de nous dire jusqu'à quel point la façon dont les nazis se sont conduits en Allemagne était insensée. Ma vision des choses serait plutôt qu'il nous montre jusqu'à quel point les docteurs non juifs ont été prêts à coopérer et jusqu'à quel point les Allemands étaient prêts à croire la propagande nazie. Il ne dit pas que ces docteurs ou que le peuple allemand de l'époque étaient volontairement coopératifs, crédules ou prêts à ignorer l'évidence. Il nous laisse inférer ce fait à partir des descriptions qu'il fournit de la situation dans ces deux cas... D'où l'ironie. Il n'y aurait aucune ironie à dire tout simplement que c'est le cas : l'ironie vient des faits décrits ou démontrés (montrés) en (38) et (39), de la même façon, dans des circonstances moins tragiques, qu'en (25).

\section{Bibliographie}

Bergson, H. Le Rire, in Euvres, Paris, Presses universitaires de France, 1959.

Chomsky, N. Structures syntaxiques, Paris, Le Seuil, 1957.

Currie, G. "Why Irony is Pretence", in Nichols, S. (ed.), The Architecture of Imagination. New Essays on Pretence, Possibility, and Fiction, Oxford/New York, Oxford University Press, 2006, 111-133.

Dumarsais, C. C. Des tropes ou des différents sens, Paris, Flammarion, 1988.

Goldman, A. "Imagination and Simulation in Audience Responses to Fiction ", in Nichols, S. (ed.), The Architecture of Imagination. New Essays on Pretence, Possibility, and Fiction, Oxford/New York, Oxford University Press, 2006a, 4156.

Goldman, A.I. Simulating Minds : the Philosophy, Psychology, and Neuroscience of Mindreading, Oxford/New York, Oxford University Press, 2006b.

Grice, H. P. Studies in the Ways of Words, Harvard, Harvard University Press, 1989.

Mosse, G. L. Les racines intellectuelles du troisième Reich. La crise de l'idéologie allemande, Paris, Calmann-Lévy, 2006.

Nichols, S. (ed.) The Architecture of Imagination. New Essays on Pretence, Possibility, and Fiction, Oxford/New York, Oxford University Press, 2006.

Perrin, L. L'ironie mise en trope, Paris, Éditions Kimé, 1996.

Reboul, A. Le discours théâtral: problèmes de narratologie et de pragmatique linguistique, thèse de doctorat, E.H.E.S.S., Paris, 1984.

Recanati, F. Oratio obliqua, oratio recta. An Essay on Metarepresentation, Cambridge, MA, The MIT Press, 2000.

Searle, J. R. Speech Acts, Cambridge, Cambridge University Press, 1969. 
Sperber, D. et D. Wilson. "Irony and the Use-Mention Distinction », in Cole, P. (ed.), Radical Pragmatics, New York, Academic Press, 1981, 295-318.

- Relevance: Communication and Cognition, Oxford, Basil Blackwell (2nd edition), 1995.

Walton, K. L. Mimesis as Make-Believe, On the Foundations of the Representational Arts, Cambridge, Mass., Harvard University Press, 1990.

Wilson, D. et R. Carston. "Metaphor, Relevance and the 'Emergent Property' Issue ", in Mind \& Language, 21/3, 2006, 404-433.

\section{Bibliographie des exemples}

Assouline, P. Gaston Gallimard: Un demi-siècle d'édition française, Paris, Balland,1984. France, A. L'île des pingouins, Genève, Amis du livre, 1955.

Kraus, K. Troisième nuit de Walpurgis, Marseille, Agone, 2005.

La Rochefoucauld. Maximes.

Molière. Tartuffe.

Stendhal. De l'amour, Paris, Editions Nilsson.

Stendhal. Lucien Leuwen, Paris, Gallimard, Folio, 1973.

Stendhal. Le Rouge et le Noir in Euvres romanesques complètes I, Paris, Gallimard, Bibliothèque de la Pléiade, 2005.

Thackeray, W.M. Barry Lindon, Harmondsworth, Penguin Books, 1975.

Voltaire. Le Taureau blanc, in Romans et contes II, Paris, Gallimard, Folio,1992.

- L'Homme aux quarante écus, in Romans et contes II, Paris, Gallimard, Folio, 1992.

—. Candide ou l'Optimiste, in Romans et contes II, Paris, Gallimard, Folio, 1992. 